\title{
ARTICLE
}

Acute myeloid leukemia

\section{RIG-I-based immunotherapy enhances survival in preclinical AML models and sensitizes AML cells to checkpoint blockade}

\author{
Michael Ruzicka ${ }^{1}$ - Lars M. Koenig $\mathbb{1}^{1,2}$. Simone Formisano ${ }^{1}$ Daniel F. R. Boehmer ${ }^{1}$ Binje Vick $\mathbb{D}^{3} \cdot$ Eva-M. Heuer ${ }^{1}$. \\ Hanna Meinl ${ }^{1}$ - Lorenz Kocheise $\mathbb{D}^{1}$ - Marcus Zeitlhöfler ${ }^{1} \cdot$ Julia Ahlfeld $^{1,2} \cdot$ Sebastian Kobold $^{1} \cdot$ Stefan Endres $^{1,2}$. \\ Marion Subklewe ${ }^{4,5,6} \cdot$ Peter Duewell $\mathbb{D}^{1} \cdot$ Max Schnurr ${ }^{1} \cdot{\text { Irmela Jeremias } \mathbb{D}^{3,6,7} \cdot \text { Felix S. Lichtenegger }}^{1,4,5}$. \\ Simon Rothenfusser (iD ${ }^{1,2}$
}

Received: 12 April 2019 / Revised: 14 October 2019 / Accepted: 3 November 2019 / Published online: 18 November 2019

(c) The Author(s) 2019. This article is published with open access

\begin{abstract}
Retinoic acid-inducible gene-I (RIG-I) is a cytoplasmic immune receptor sensing viral RNA. It triggers the release of type I interferons (IFN) and proinflammatory cytokines inducing an adaptive cellular immune response. We investigated the therapeutic potential of systemic RIG-I activation by short 5'-triphosphate-modified RNA (ppp-RNA) for the treatment of acute myeloid leukemia (AML) in the syngeneic murine C1498 AML tumor model. ppp-RNA treatment significantly reduced tumor burden, delayed disease onset and led to complete remission including immunological memory formation in a substantial proportion of animals. Therapy-induced tumor rejection was dependent on $\mathrm{CD}^{+}$and $\mathrm{CD} 8^{+} \mathrm{T}$ cells, but not on NK or B cells, and relied on intact IFN and mitochondrial antiviral signaling protein (MAVS) signaling in the host. Interestingly, ppp-RNA treatment induced programmed death ligand 1 (PD-L1) expression on AML cells and established therapeutic sensitivity to anti-PD-1 checkpoint blockade in vivo. In immune-reconstituted humanized mice, ppp-RNA treatment reduced the number of patient-derived xenografted (PDX) AML cells in blood and bone marrow while concomitantly enhancing $\mathrm{CD}^{+} \mathrm{T}$ cell counts in the respective tissues. Due to its ability to establish a state of full remission and immunological memory, our findings show that ppp-RNA treatment is a promising strategy for the immunotherapy of AML.
\end{abstract}

These author contributed equally: Michael Ruzicka and Lars M. Koenig as well as Felix S. Lichtenegger and Simon Rothenfusser

Supplementary information The online version of this article (https:// doi.org/10.1038/s41375-019-0639-x) contains supplementary material, which is available to authorized users.

Simon Rothenfusser

simon.rothenfusser@med.uni-muenchen.de

1 Center of Integrated Protein Science Munich (CIPS-M) and Division of Clinical Pharmacology, University Hospital, LMU Munich, Munich, Germany

2 Einheit für Klinische Pharmakologie (EKLiP), Helmholtz Zentrum München, German Research Center for Environmental Health (HMGU), Neuherberg, Germany

3 Research Unit Apoptosis in Hematopoietic Stem Cells, Helmholtz Zentrum München, German Research Center for Environmental Health (HMGU), Neuherberg, Germany

\section{Introduction}

Acute myeloid leukemia (AML) is a clonal disease of hematopoietic precursor cells. Despite some improvements in treatment and outcome parameters over the past decades, the prognosis of the disease is still dismal. High risk of relapse despite initial complete remission is the major reason for poor survival rates. This is due to chemorefractory residual leukemic stem cells remaining after intensive

4 Department of Medicine III, University Hospital, LMU Munich, Munich, Germany

5 Laboratory for Translational Cancer Immunology, Gene Center, LMU Munich, Munich, Germany

6 German Cancer Consortium (DKTK), partner site Munich, Munich, Germany

7 Department of Pediatrics, Dr. von Hauner Children's Hospital, Ludwig Maximilian University, Munich, Germany 
chemotherapy [1]. Hematopoietic stem cell transplantation (HSCT) is a potentially curative treatment option based on the graft-versus-leukemia (GvL) effect of allogeneic T cells [2]. However, HSCT is associated with high morbidity and mortality and is thereforee not a treatment option for many AML patients. Particularly for this patient population, there is a high need for novel therapeutic options including new postremission treatments. Alternative strategies to recruit the immune system for eradication of the disease are studied intensively including antibody-drug conjugates, $\mathrm{T}$ cellrecruiting antibody constructs, vaccination concepts, checkpoint inhibitors, and chimeric antigen receptor $\mathrm{T}$ cells, although their clinical development for AML still lags behind compared to other malignant diseases [3].

RIG-I-like receptor ligands have been used as a promising strategy for the treatment of solid malignancies including melanoma $[4,5]$, pancreatic cancer [6] and breast cancer [7] in preclinical models and just recently qualified for a combined phase I/II study in patients with advanced solid tumors (NCT03065023). Serving as a cytoplasmic antiviral pattern recognition receptor, RIG-I senses short double-stranded RNAs with an uncapped 5'-triphosphate moiety (ppp-RNA), a common motif found in viral RNAs [8-12]. Upon binding to viral RNA, RIG-I interacts with the mitochondrial antiviral signaling protein (MAVS) [13-15]. MAVS, once activated, assembles a downstream signaling complex culminating in the activation of nuclear factor kappa-light-chain-enhancer of activated B cells (NF- $\mathrm{kB}$ ) and interferon regulatory factor 3/7 (IRF3/IRF7), resulting in the release of type I interferons (IFN) and proinflammatory cytokines (reviewed in Chow et al. [16]). Furthermore, ppp-RNA has been shown to induce an immunogenic form of cell death (ICD) in different tumor entities [7, 17-19]. The cytokine release combined with direct sensing of viral RNA by immune cells leads to an adaptive cellular immune response directed against infected cells [4]. By applying an exogenous short ppp-RNA, a viral infection can be mimicked, allowing direction of the immune response towards otherwise altered or potentially harmful targets, such as cancerous cells. While this approach has shown survival benefits in different solid tumor models $[4,6,7,20]$, the treatment efficacy of RIG-I ligands for non-solid tumors in vivo remains elusive. We therefore investigated the potential of ppp-RNA therapy for AML as an example of a hematological malignancy.

RIG-I was first identified as a gene induced by retinoic acid in a promyelocytic leukemia cell line [21] and its expression was described to restrain leukemic activity in AML blasts [22]. Since mainly affecting the bone marrow and blood, we expected AML to be more susceptible to intravenous (i.v.) treatment with ppp-RNA, subsequent systemic cytokine responses and immune cell-mediated cytotoxicity than solid tumors. In addition, we hypothesized that the prominent role of IFN release in ppp-RNA therapy and the subsequent upregulation of programmed death ligand 1 (PD-L1) [23] expression holds potential for combining ppp-RNA with anti-PD-1 receptor antibodies. In recent years, blockade of the inhibitory PD-1/PD-L1 axis resulting in increased cytotoxic activity of $\mathrm{T}$ cells was investigated in numerous malignancies with great success [24-27]. However, so far PD-1/PDL1 blockade has only shown moderate success as a monotherapy for AML in clinical settings [3] and in the C1498 mouse model [28]. Here, we explore the responsiveness of AML to ppp-RNA treatment alone and in combination with anti-PD-1 blocking antibodies in the murine syngeneic C1498 AML model and in immune-reconstituted humanized mice with patientderived xenografted (PDX) AML cells.

\section{Materials and methods}

PDX AML cells were established as described previously [29] and a detailed description of the used mouse models, cell lines, reagents, and methods is provided as part of the supplementary information.

\section{Results}

\section{Systemically administered ppp-RNA decreases AML burden in vivo, delays $A M L$ progression and leads to long-term survival in a murine syngeneic mouse model}

To asses the in vivo efficacy of RIG-I-based immunotherapy of AML we utilized the C1498 model, a murine AML cell line on C57BL/6 background classified as acute myelomonocytic leukemia [30], which was implanted in immune competent syngeneic mice. It is characterized by highly aggressive growth with regular infiltration of the bone marrow, blood, spleen, liver, lung, and ovaries (data not shown). After inoculation, mice appear asymptomatic for 15-20 days. First signs of disease are commonly followed by rapid progression requiring the sacrifice of the animals. In order to investigate the overall therapeutic potential of systemic ppp-RNA treatment with regard to AML, we inoculated C57BL/6 mice with $1 \times 10^{6}$ GFP expressing C1498 AML cells (C1498-GFP) via tail vein injection. ppp-RNA was complexed with in vivo-jetPEI in order to protect it from nuclease digestion and enable cytoplasmic delivery of ppp-RNA. Fifty micrograms of complexed ppp-RNA were administered intravenously on days $3,7,10$, and 14 after tumor inoculation (as depicted in Fig. 1a). Mice were sacrificed on day 17, and the tumor burden in various organs was assessed (Fig. 1b). Significant 
A

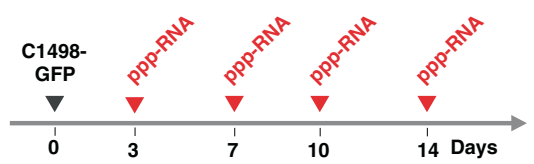

B

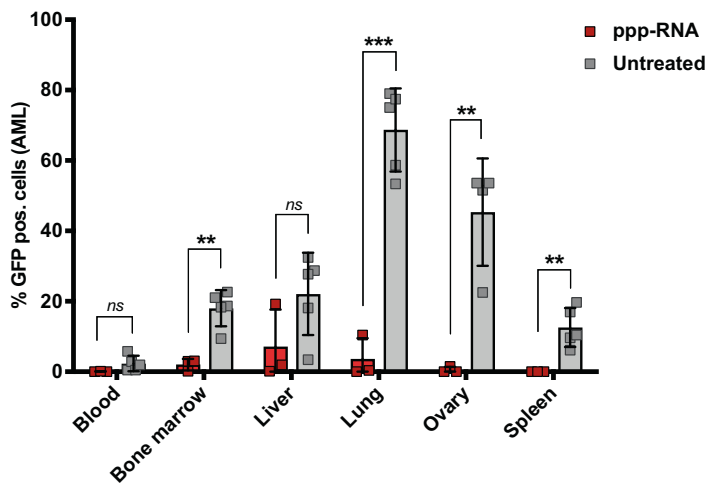

Fig. 1 Systemic ppp-RNA treatment in C1498-GFP tumor bearing mice. a Therapy scheme for in vivo experiments in the syngeneic C1498-GFP AML model. AML was induced by injecting $10^{6} \mathrm{C} 1498-$ GFP AML cells into the tail vein. On days 3, 7, 10 and 14, mice were treated with $50 \mu \mathrm{g}$ ppp-RNA i.v. b ppp-RNA treated (bar charts indicate mean of $n=3$ with SEM of a single experiment) and untreated (bar charts indicate mean of $n=5$ with SEM) C57BL/6 mice were sacrificed on day 17. Single cell suspensions of blood, bone
C

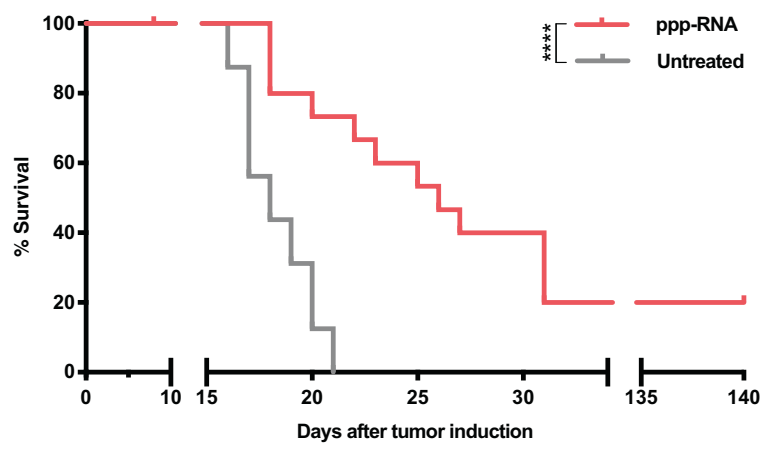

marrow, livers, lungs, ovaries, and spleens were analyzed by flow cytometry determining the fraction of $\mathrm{GFP}^{+}$cells (AML cells). Statistical significance was determined using the Student's $t$ test with comparisons indicated by brackets. c C1498-GFP AML was induced in C57BL/6 mice ( $n=16$ per group derived from three independent experiments) and ppp-RNA therapy was applied according to the scheme in a. Survival data were plotted in a Kaplan-Meier survival curve and statistical significance was calculated with the log-rank test reduction of tumor mass was observed in bone marrow $\left(2.1 \%\right.$ vs $18.1 \% \mathrm{GFP}^{+}$cells for ppp-RNA treated $(n=3)$ vs untreated $(n=5), p=0.002)$, lungs $\left(3.6 \%\right.$ vs $68.8 \% \mathrm{GFP}^{+}$ cells for ppp-RNA treated $(n=3)$ vs untreated mice $(n=5)$, $p<0.001)$, ovaries $\left(0,5 \%\right.$ vs $45,3 \% \mathrm{GFP}^{+}$cells for pppRNA treated $(n=3)$ vs untreated mice $(n=4), p=0.004)$, and spleens $\left(0.03 \%\right.$ vs $12.6 \% \mathrm{GFP}^{+}$cells for ppp-RNA treated $(n=3)$ vs untreated mice $(n=5), p=0.009)$.

We next evaluated the effects of ppp-RNA treatment on the survival of the mice. C1498-GFP AML-bearing mice were treated as described above and animals were sacrificed at signs of disease such as weight loss, ascites, apathy and/ or development of visible tumors and survival data were plotted in a Kaplan-Meier survival curve (Fig. 1c). Beyond a significantly delayed onset of symptomatic AML disease (median survival 26 days vs. 18 days with $p<0.0001$ for ppp-RNA treated $(n=16)$ versus untreated mice $(n=16)$ ), ppp-RNA treatment led to long-term remission in 3 out of $16(19 \%)$ of the treated animals, demonstrating the potential of systemic RIG-I activation in the therapy of AML.

\section{Treatment efficacy depends on $\mathrm{CD}^{+}$and $\mathrm{CD8}^{+}$ $T$ cells, but not $B$ and NK (natural killer) cells}

Since the C1498-GFP AML cell line resists transfection with most commercially available RNA transfection reagents in vitro and is thus difficult to target directly with
ppp-RNA, it was of great interest to us to narrow down the mechanisms responsible for the observed tumor rejection. In order to discriminate between a direct cytotoxic effect of ppp-RNA on the tumor cells and immune cell-mediated responses, immune incompetent NSG mice lacking T, B, and NK cells were inoculated with C1498-GFP AML cells. ppp-RNA treatment was given on days 3, 6, 9, and 12 . Both treated and untreated animals died on day 13 (Fig. 2a) and no significant differences in tumor burden were detected between the two groups via flow cytometry (Supplementary Fig. S1), demonstrating a prominent role of the adaptive immune system in the ppp-RNA-mediated treatment effect.

Based on these findings we next asked which immune cell types were necessary for the survival benefit we observed in treated wild-type mice. Therefore, we specifically depleted $\mathrm{CD} 4^{+}, \mathrm{CD} 8^{+} \mathrm{T}$ cells, $\mathrm{CD} 19^{+} \mathrm{B}$ or NK1.1 $1^{+}$ NK cells (Supplementary Fig. S2) and compared the treatment success. While $\mathrm{CD}^{+}(p=0.003)$ and $\mathrm{CD}^{+} \mathrm{T}$ cell $(p=0.018)$ depletion resulted in significant loss of therapeutic efficacy (Fig. 2a), CD19 $(p=0.401)$ and NK1.1 $(p=0.376)$ depletion remained without significant effect compared to the respective isotype controls (Fig. 2b).

Taken together, these data show that the treatment efficacy of ppp-RNA is dependent on adaptive immunity with $\mathrm{CD}^{+}$and $\mathrm{CD}^{+} \mathrm{T}$ cells being essential mediators of the antitumoral immune response. 
A
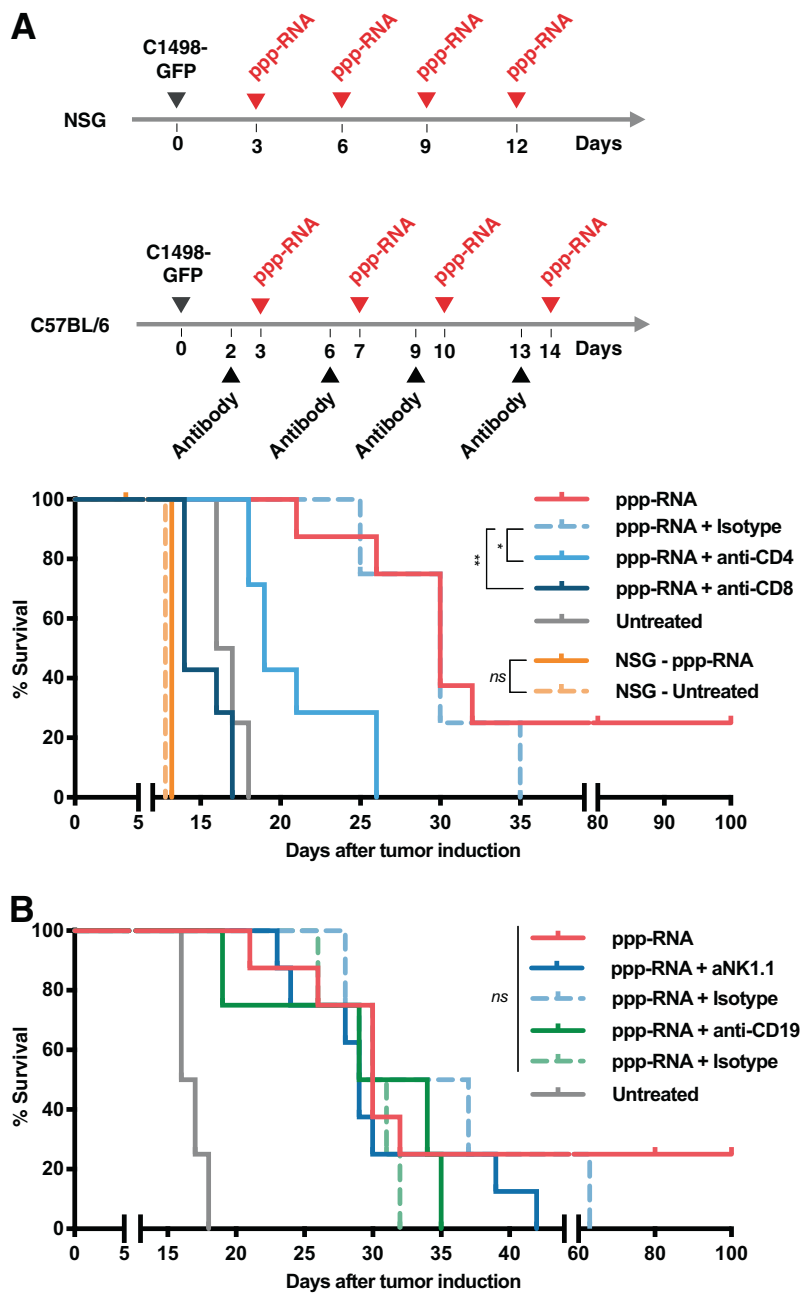

Fig. 2 ppp-RNA induced tumor rejection is mediated by cellular immunity. a NSG mice and C57BL/6 mice $(\mathbf{a}, \mathbf{b})$ were inoculated with C1498-GFP AML cells and therapy was applied according to the schemes depicted in a. NSG mice ( $n=5$ for ppp-RNA treated, $n=4$ for untreated, both derived from one experiment), C57BL/6 mice $(n=$ 8 per group for ppp-RNA treated, untreated and ppp-RNA treated + NK1.1 depleted C57BL/6 mice; $n=7$ for ppp-RNA treated+CD4/ CD8 depleted C57BL/6 mice. Data derive from two independent experiments; $n=4$ for ppp-RNA treated + CD19 depleted C57BL/6 mice, these data derive from a single experiment). Depleting antibodies were administered as described in the materials and methods section. Corresponding isotype controls were tested in a total of $n=4$ mice per group in a single experiment. Survival data were plotted in two Kaplan-Meier survival curves. $P$ values of immune cell depleted groups compared to respective isotype controls were calculated using the log-rank test: $p=0.018$ for $\mathrm{CD} 4, p=0.003$ for CD8, $p=0.376$ for NK1.1, $p=0.401$ for CD19 depletion

\section{ppp-RNA induced long-term survival in AML-bearing mice depends on systemic MAVS and type I IFN signaling}

Apart from RIG-I, various other pattern recognition receptors are known to directly or indirectly sense short doublestranded and hairpin RNAs [31], resulting in type I IFN induction. To determine the contribution of off-target effects on the therapeutic outcome of ppp-RNA treatment in the C1498 model and to evaluate the role of RIG-I signaling in the host, we compared the effects of ppp-RNA in wild type (WT), MAVS- and IFNAR1-deficient mice treated according to the scheme depicted in Fig. 3a. To estimate the immunostimulatory activity of ppp-RNA in the respective mice, serum CXCL10 levels were measured as a surrogate marker for type I IFN release after the first and fourth treatment. ppp-RNA therapy in WT and $\mathrm{Mavs}^{-/}$ mice resulted in comparable serum levels of CXCL10 four hours after the first treatment $(p=0.986$ for WT versus Mavs $^{-/-}$mice; Fig. 3a), while in Ifnarl $^{-/-}$mice no CXCL10 was measured after either treatment. Interestingly, CXCL10 levels four hours upon the fourth ppp-RNA treatment in $\mathrm{Mavs}^{-/-}$mice dropped below detection limit while CXCL10 levels measured in WT mice remained constant $(p=0.469$ for first vs. fourth treatment in WT mice, $p=0.001$ for first vs. fourth treatment in $\mathrm{Mavs}^{-/-}$ mice and $p<0.001$ for WT vs. Mavs $^{-1-}$ mice after fourth treatment; Fig. 3a).

In Ifnarl ${ }^{-1-}$ mice, ppp-RNA treatment did not lead to a survival benefit compared to untreated animals $(p=0.073$; Fig. 3b), indicating a central role of intact type I IFN signaling for the therapeutic effect. We asked the question whether type I IFN may solely mediate the therapeutic effect of ppp-RNA in this particular model of AML. Therefore, therapeutic doses of murine IFN alpha were administered i.p. to C1498-GFP AML tumor bearing WT mice on days 3, 7,10 , and 14 . The therapeutic efficacy of ppp-RNA failed to be recapitulated by IFN alpha alone $(p=0.352$ for IFN alpha treated vs. untreated mice; Fig. 3c), suggesting that type I IFN is essential, but on its own insufficient for the immune response initiated by ppp-RNA treatment.

In $\mathrm{Mavs}^{-/-}$mice, ppp-RNA therapy prolonged diseasefree survival despite disrupted RIG-I signaling $(p=0.0193$ for ppp-RNA treated vs. untreated mice; Fig. 3d). However, the effect was less pronounced than in WT mice (hazard ratios for ppp-RNA treated vs. untreated mice: 0.229 in $\mathrm{Mavs}^{-1-}$ vs. 0.113 in WT mice). Of note, no long-term survival was observed in $\mathrm{Mavs}^{-/-}$mice in the treated group.

The results demonstrate that ppp-RNA induced tumor rejection in this AML model is mediated by, but not limited to effects of type I IFN release. Despite CXCL10 levels being comparable after the first ppp-RNA treatment in WT and $\mathrm{Mavs}^{-1-}$ mice, intact RIG-I signaling via MAVS in the host seems to be essential particularly for repeated IFN induction and long-term survival in ppp-RNA treated animals.

\section{ppp-RNA treatment induces immunological memory}

Next, we evaluated if a long-lasting immunological memory was established in ppp-RNA-treated mice that had survived 
A

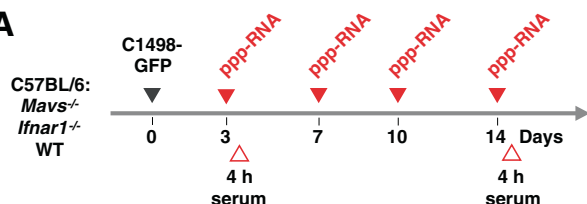

B
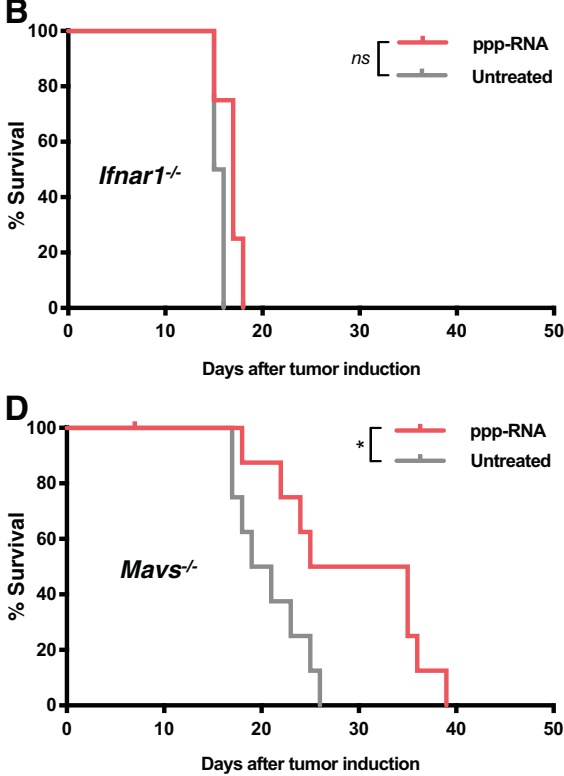

Fig. 3 ppp-RNA treatment depends on intact IFN alpha signaling. a As depicted in the schematic C57BL/6 WT, Mavs $^{-1-}$ and Ifnarl ${ }^{-/-}$mice were treated with $50 \mu \mathrm{g}$ of ppp-RNA on days 3, 7, 10, and 14 after inoculation with C1498-GFP AML cells. Blood was drawn after the first (day 3) and fourth (day 14) treatment, and levels of murine CXCL10 were measured via ELISA. Each symbol represents a single mouse and error bars indicate SD. Statistical differences between genotypes at one time point were determined by one-way ANOVA with Tukey's post-hoc test. b Ifnarl $^{-/-}$mice ( $n=4$ per group) were inoculated with C1498-GFP AML cells and treated according to the scheme depicted in a. Survival data were plotted in a Kaplan-Meier

the AML challenge. Surviving mice were rechallenged with C1498-GFP AML cells on day 85-110 after the first AML inoculation and compared to tumor-inoculated control animals. Survivor mice withstood the AML rechallenge in all cases ( $n=7$ for AML surviving vs. $n=10$ for tumor-naive mice, $p<0.0001$; Fig. 4a).

In order to further investigate the role of $\mathrm{CD}^{+} \mathrm{T}$ cells in the memory responses observed, we adoptively transferred $\mathrm{CD}^{+} \mathrm{T}$ cells from survivor mice into therapy-naive WT recipient C57BL/6 mice $12 \mathrm{~h}$ prior to inoculation with C1498-GFP AML cells. CD8 ${ }^{+}$T cells from healthy C57BL/ 6 mice served as control. Significant delay of disease onset was observed in mice treated with $\mathrm{CD} 8^{+} \mathrm{T}$ cells derived from ppp-RNA treated, AML surviving mice compared to tumor-naive donors ( $p=0.007$; Fig. $4 \mathrm{~b})$, indicating a central role of $\mathrm{CD}^{+} \mathrm{T}$ cells as mediators of the immunological memory established.
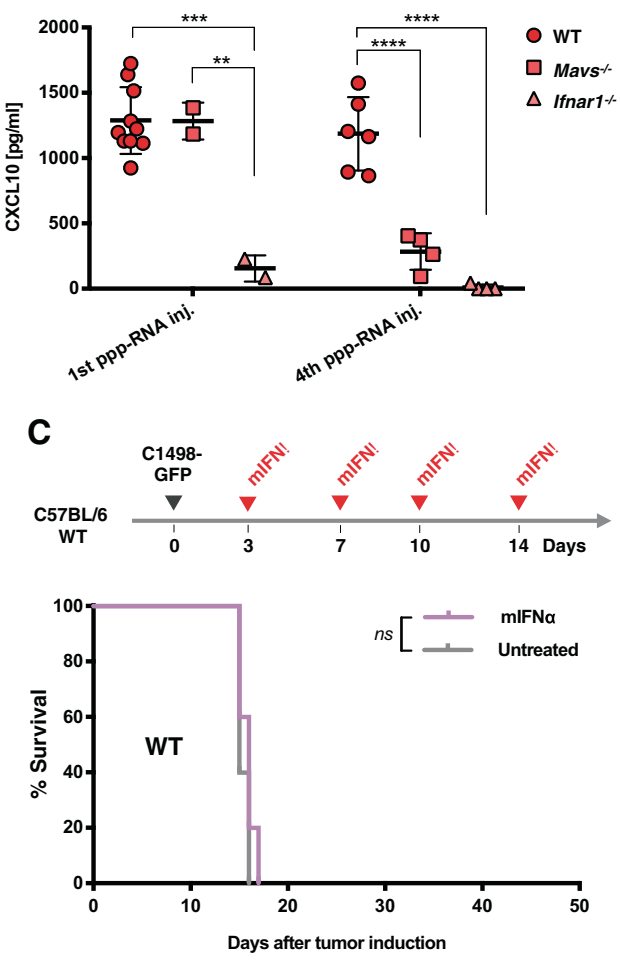

survival curve. $p=0.073$ for ppp-RNA versus untreated mice. c C1498-GFP AML-bearing C57BL/6 wild type mice $(n=10)$ were randomized into two groups, of which one received $5 \times 10^{4} \mathrm{IU}$ murine IFN alpha $(\mathrm{mIFN} \alpha)$ i.p. on days 3, 7, 10, and 14 as depicted in the schematic in c. Survival data were plotted in a Kaplan-Meier survival curve. $p=0.352$ for mIFN $\alpha$ treated versus untreated mice. d C57BL/6 Mavs $^{-1-}$ ( $n=9$ per group) were treated with $50 \mu \mathrm{g}$ of ppp-RNA on days $3,7,10$, and 14 or left untreated according to the scheme depicted in a. Survival data were plotted in a Kaplan-Meier survival curve. The data shown are derived from one $(\mathbf{b}, \mathbf{c})$ or two independent $(\mathbf{d})$ experiments

\section{ppp-RNA mediated antitumor response is boosted by PD-1 checkpoint inhibition in vivo}

In vitro, we observed strong upregulation of PD-L1 on C1498-GFP AML cells upon stimulation with IFN gamma ( $p<0.001$ for IFN gamma treated vs. untreated cells; Fig. 5a). Considering the prominent role of IFN signaling involved in ppp-RNA treatment of C1498-GFP AML (Fig. 3b), we analyzed PD-L1 expression on tumor cells infiltrating lung tissue of C57BL/6 mice, as in this organ we observed the strongest effects of ppp-RNA on tumor load (see Fig. 1b). Mice were sacrificed $12 \mathrm{~h}$ after ppp-RNA injection and lung tissue was analyzed by flow cytometry, revealing significant upregulation of PD-L1 on tumor cells ( $p=0.002$; Fig. $5 b$ ). Next, we treated C1498-GFP AML-bearing C57BL/6 mice with pppRNA on days $3,7,10$, and 14, applying a suboptimal dose of $25 \mu \mathrm{g}$ RNA. In addition, $100 \mu \mathrm{g}$ of anti-PD-1 blocking 


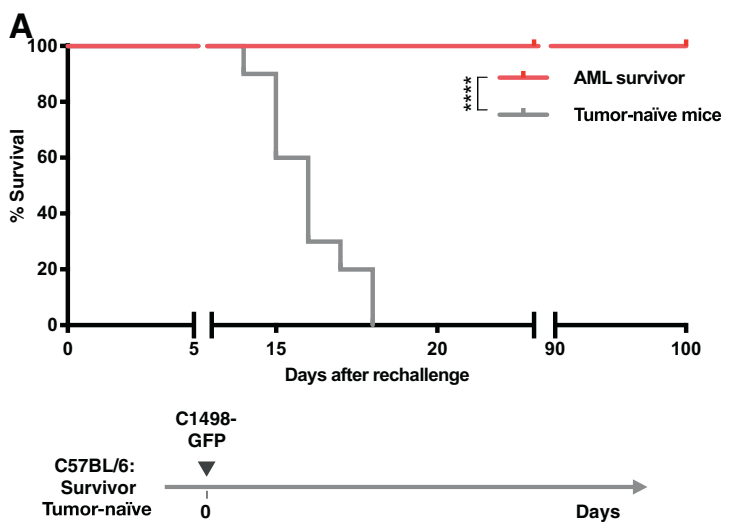

Fig. 4 Tumor rechallenge of C1498-GFP AML surviving mice and adoptive transfer of $\mathrm{CD}^{+} \mathrm{T}$ cells. a C1498-GFP AML surviving C57BL/6 mice $(n=7)$ were rechallenged with $10^{6}$ C1498-GFP AML cells. No further treatment was applied. Tumor-naive C57BL/6 mice $(n=10)$ served as controls. Survival data derived from three independent experiments were plotted in a Kaplan-Meier survival curve. b As depicted in the schematic C57BL/6 mice $(n=4)$ were treated

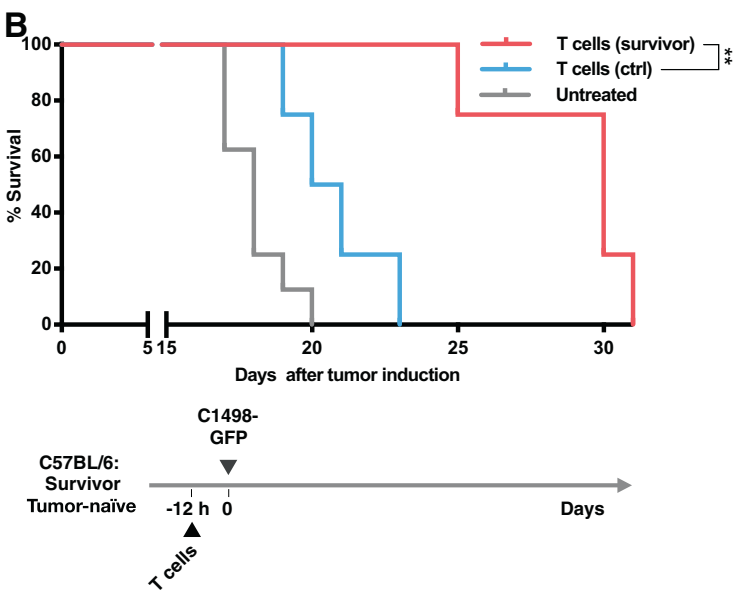

with $\mathrm{CD}^{+} \mathrm{T}$ cells from C1498-GFP AML surviving (T cells (survivor), $n=3$ ) or tumor-naive mice ( $\mathrm{T}$ cells $(\mathrm{ctrl}), n=5)$, respectively. Untreated mice $(n=8)$ served as controls. C1498-GFP AML was induced $12 \mathrm{~h}$ later in all three groups. T cells were isolated as described in the "Materials and methods" section. Survival data derived from two independent experiments were plotted in a Kaplan-Meier survival curve. Significance was calculated using log-rank test

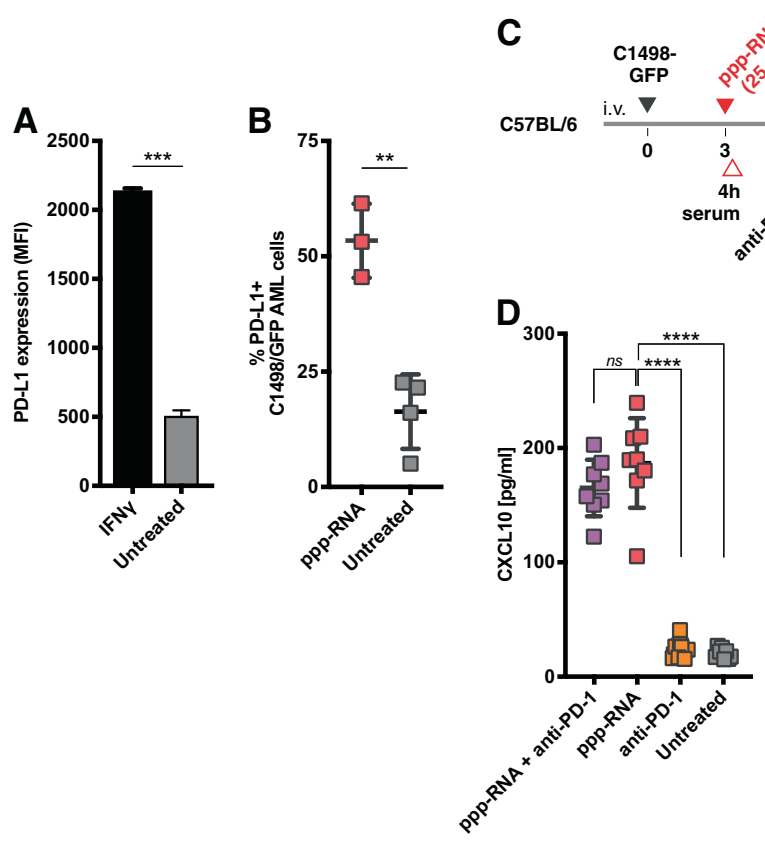

Fig. 5 ppp-RNA treatment primes AML cells for anti-PD-1 checkpoint inhibition. a $2.5 \times 10^{5} \mathrm{C} 1498$-GFP AML cells were seeded in six-well format and treated with interferon gamma. PD-L1 expression was determined by flow cytometry $72 \mathrm{~h}$ after stimulation. b C1498-GFP AML-bearing C57BL/6 mice received three treatments of $50 \mu \mathrm{g}$ pppRNA on days 8,11 , and 14 after tumor induction. Twelve hours upon the last treatment (day 15), mice were sacrificed and single cell suspensions of lung tissue were analyzed by flow cytometry, determining PD-L1 expression on GFP ${ }^{+}$AML cells. c, d C57BL/6 mice $(n=13$ per group derived from two independent experiments) were inoculated

antibody were administered i.p. on days 6, 9, and 13 (as depicted in Fig. 5c). Serum levels of murine CXCL10 were measured $4 \mathrm{~h}$ after the first ppp-RNA treatment on day 3
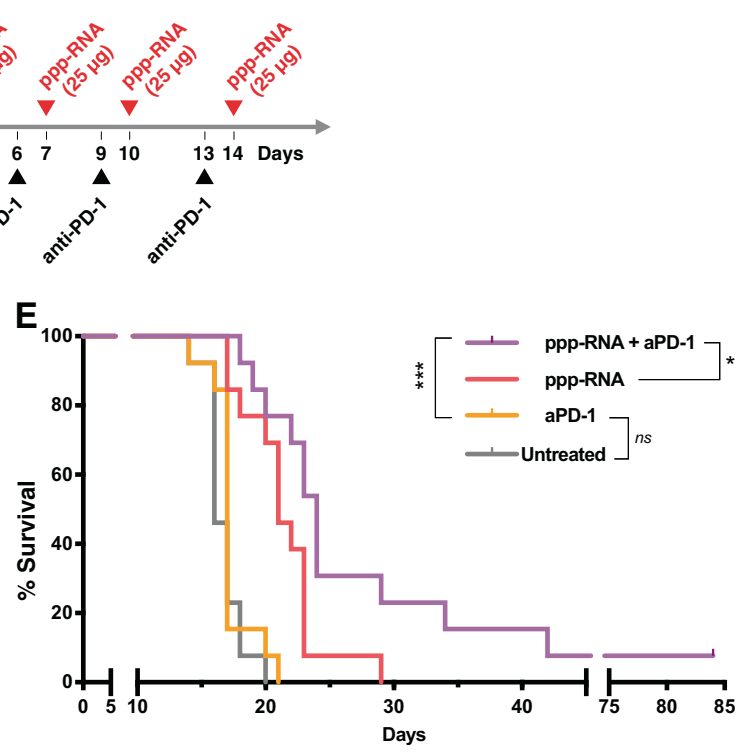

with C1498-GFP AML cells on day 0 and treated with $25 \mu \mathrm{g}$ of pppRNA on days $3,7,10$, and 14. Hundred micrograms of anti-PD-1 antibody was injected i.p. on days 6, 9, and 13. Levels of murine CXCL10 were determined by ELISA in blood serum obtained $4 \mathrm{~h}$ after the first treatment with $25 \mu \mathrm{g}$ of ppp-RNA ( $n=8$ per group) c. Survival data were plotted in a Kaplan-Meier survival curve (e). Statistical significance was determined by the Student's $t$ test $(\mathbf{a}, \mathbf{b})$, one-way ANOVA with the Tukey's post-hoc test (c) and the log-rank test (e)

(Fig. 5d). Treatment with anti-PD-1 antibody had no effect on CXCL10 serum levels. However, the combination of pppRNA and anti-PD-1 checkpoint blockade was superior to 
ppp-RNA or anti-PD-1 antibody alone ( $p=0.02$ and $p<$ 0.001 , respectively; Fig. 5e) in regard to survival (see also Supplementary Fig. S3A-C). Treatment with the anti-PD-1 antibody only remained without any antitumor effect. Thus, ppp-RNA sensitized AML cells to concomitant checkpoint inhibition leading to superior tumor control. Interestingly, CXCL10 serum levels after the first ppp-RNA treatment (Fig. 5d) correlated with survival time in the ppp-RNA only treatment group $(r=0,75 ; 95 \%$ confidence interval: 0.098 to $0.951 ; p=0,031 ; n=8)$ an observation not seen in the group treated with both, ppp-RNA and anti-PD-1.

\section{Validation of ppp-RNA treatment efficacy in a humanized mouse model of AML}

We approached the potential of ppp-RNA-based immunotherapy for clinical translation by testing a genetically diverse panel of five human AML cell lines (MV4-11, OCIAML3, Molm-13, PL-21 and THP-1) and five patientderived (PDX) AML blasts (AML-372, AML-388, AML491, AML-896, AML-981 (see Supplementary Table S1)) for their responses to ppp-RNA ex vivo. These diverse AML cells covering common mutations occurring in human AML all responded to ppp-RNA with the production of CXCL10, the upregulation of MHC-class I, PD-L1 and to variable degrees with the upregulation of FAS and the induction of cell death (see Supplementary Fig. S4). These data confirm that human AML cells have an intact RIG-I signaling pathway and that triggering this pathway induces a measurable but limited direct cytotoxic effect in human AML cells. In addition they suggest that, reminiscent of the effects seen in the C1489 mouse model, ppp-RNA might sensitize human AML cells to $\mathrm{T}$ cell-mediated cell death (via enhanced MHC-class I/TCR recognition and Fas/Fasligand interaction) and to checkpoint blockade of the PD-1/ PD-L1 axis. However, the C1489 model has clearly shown that in vivo the direct cytotoxic effect of ppp-RNA on AML cells alone does not explain the therapeutic benefit of this treatment and that the potential of ppp-RNA treatment can only be seen in the presence of an intact T-cell response. We therefore designed an immune-reconstituted humanized mouse model of AML using PDX AML cells for further validation. NSG mice were inoculated with $4.5 \times 10^{5} \mathrm{PDX}$ AML-491 cells via tail vein injection, and tumor growth was monitored via flow cytometry in peripheral blood. An average tumor load of $51 \%$ in peripheral blood was detected on day 52 (see Supplementary Fig. S5) and all animals received $1 \times 10^{7}$ human PBMCs from a healthy, partlyHLA-matched donor via tail vein injection. Three doses of $50 \mu \mathrm{g}$ ppp-RNA were given on days 53, 56, and 59. Mice were sacrificed on day 60 and AML loads as well as immune cell numbers in peripheral blood and bone marrow were determined by flow cytometry (Fig. 6a, b, respectively). Lower tumor burdens were detected in peripheral blood $(p<0.001)$ and as a trend in bone marrow (ns, $p=$ 0.071) in ppp-RNA treated compared to untreated mice. Human $\mathrm{CD}^{+} \mathrm{T}$ cells were found at higher numbers in the treatment group in both compartments $(p<0.0001$ for blood, $p=0.005$ for bone marrow), suggesting enhanced immune cell expansion after treatment.

\section{Discussion}

Targeting RIG-I with ppp-RNA has been described in preclinical studies as a promising strategy in the treatment
A

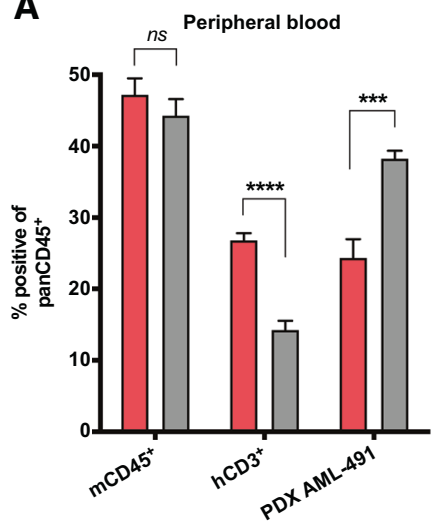

Fig. 6 Efficacy of ppp-RNA in a humanized mouse model of AML. Twelve NSG mice were inoculated with $4.5 \times 10^{5}$ PDX AML-491 cells i.v. on day 0 and $10^{7}$ human PBMCs were injected on day 52 . ppp-RNA treatment was given on days 53, 56, and 59. Mice were sacrificed $12 \mathrm{~h}$ after the last treatment on day 60. Single cell suspensions of peripheral blood (a) and bone marrow (b) were analyzed by flow cytometry, determining levels of murine CD45 positive

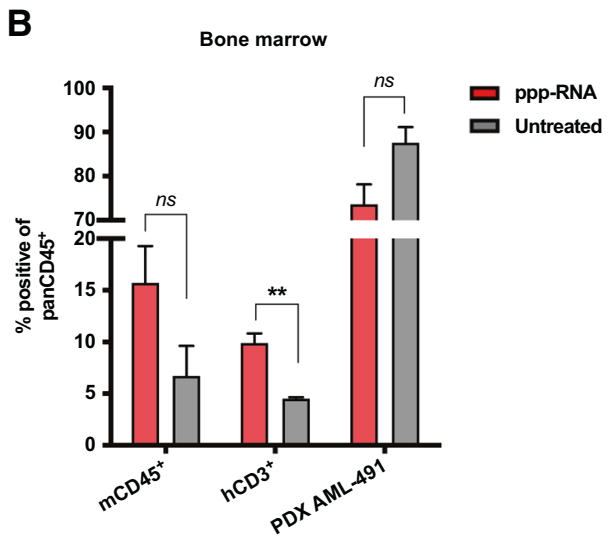

$\left(\mathrm{mCD} 45^{+}\right)$and human $\mathrm{CD} 3$ positive cells $\left(\mathrm{hCD}^{+}\right)$relating to murine plus human $\mathrm{CD} 45$ positive (pan $\mathrm{hCD} 45^{+}$) cells. Tumor burdens were determined by the detection of mCherry positive cells. Bar charts depict mean values plus SEM of $n=6$ (a) and $n=3$ (b). Statistical significance was determined using a Student's $t$ test with comparisons indicated by brackets 
of various solid tumors $[4,5,7,17,20,32]$. However, no data exist suggesting similar efficacy for hematologic malignancies. Here, we show that systemic activation of RIG-I using ppp-RNA not only extends survival, but also leads to full remission in some mice bearing disseminated syngeneic AML tumors and establishes an immunological memory in surviving animals.

Immunotherapy using ppp-RNA has been shown to rely on two mechanisms, namely a direct cytotoxic effect on the tumor cells $[5,17,18]$ and the induction of a cellular antitumoral immune response. While the former mechanism has previously been described as RIG-I-dependent apoptosis induction in a human xenograft melanoma mouse model [17] and different in vitro models, the complete absence of a treatment benefit on disseminated AML tumors in NSG mice indicates no direct cytotoxic or cytostatic effect of ppp-RNA treatment on AML cells in our model. Moreover, the results indicate that the cell-intrinsic antileukemic effect of RIG-I via the AKT and mTOR pathway that has previously been proposed [22] does not play a role in our model. In contrast, rejection of leukemic cells seems to be mediated by the induction of tumor-cell specific cellular immunity, which is consistent with the mode of action of ppp-RNA enhancing cross-presentation by antigen-presenting cells and inducing an adaptive antitumoral response [33]. We further investigated the contribution of individual immune cell types using depleting antibodies in WT mice. We identified $\mathrm{CD}^{+}$and $\mathrm{CD}^{+} \mathrm{T}$ cells to mediate the treatment response while $\mathrm{B}$ and NK cells were dispensable. NK cells as mediators of pppRNA-induced antitumor responses have been described for B16 melanoma cells [4], but were also dispensable in models of pancreatic cancer [6] and hepatocellular carcinoma (unpublished data), suggesting that the mechanisms of pppRNA-induced tumor rejection vary depending on the tumor entity and vulnerability. RIG-I signaling via MAVS in the host plays a crucial role for ppp-RNA treatment outcome. Overall survival in $\mathrm{Mavs}^{-/-}$mice was inferior to survival in WT mice, while long-term survival and memory formation was observed in WT mice exclusively. The residual effects we observed are possibly linked to Toll-like receptor (TLR) 7, which is known to sense single-stranded RNA and may be activated by ppp-RNA. In fact, previous studies show that repeated systemic activation of TLR7 leads to desensitization of the receptor [34],explaining the diminished CXCL10 induction after repeated treatment with ppp-RNA in $\mathrm{Mavs}^{-/-}$ mice compared to WT mice (Fig. 3a). Nevertheless, the outcome of ppp-RNA treatment has previously been shown to be unimpaired in $T l r 7^{-/-}$mice [32], again underlining that MAVS is the critical receptor to achieve long-term remission, independently of potential off-target effects. A central factor of the induction of an antitumoral immune response is type I IFN. Consistent with previously published results, we identified intact IFNAR signaling in the host organism as a key factor for treatment efficacy in the AML model. Disseminated leukemia is known for its inability to induce type I IFN in contrast to subcutaneously grown leukemic tumors, thus failing to activate cellular antitumor immunity [35]. Elevated levels of the interferon-inducible chemokine CXCL10 after treatment suggest that ppp-RNA therapy does induce the release of type I IFN, which in turn paves the way for a cellular immune response as described above. Importantly, IFN treatment alone fails to induce leukemia rejection, emphasizing a ppp-RNA specific effect that initiates an adaptive immune response in a multistep process in which type I IFN plays a critical part [36].

The induction of an immunological long-term memory by ppp-RNA therapy makes this approach particularly interesting for the treatment of AML as it holds potential to prevent relapse. We discovered that all mice surviving C1498-GFP AML after ppp-RNA treatment established an immunological memory, protecting them from a rechallenge with C1498-GFP AML cells. Moreover, $\mathrm{CD}^{+} \mathrm{T}$ cells adoptively transferred from AML surviving mice into AML-bearing WT mice extended survival significantly, but could not induce long-term remission. The partial protection is likely explained by the fact that transferred $\mathrm{T}$ cells most likely contained only a small fraction of antigen-specific $\mathrm{T}$ cells that in addition expanded and worked insufficiently in vivo due to the lack of cognate $\mathrm{T}$ helper cells.

Although anti-PD-1 monotherapy proved to be inefficacious in the C1498-GFP AML model, PD-1 inhibition showed augmented therapeutic effects when administered together with ppp-RNA treatment. The combination of both agents was superior in outcome to either of them applied alone, suggesting a priming role of ppp-RNA for the antibody to become therapeutically relevant. This assumption is strengthened by the finding that PD-L1 is strongly upregulated on C1498-GFP tumor cells in vivo after ppp-RNA treatment and was also induced in vitro on human AML cells (Supplementary Fig. S4). As preliminary data indicate, the synergistic nature of ppp-RNA and anti-PD-1 antibodies may hold potential for even higher survival rates if the dose used in our experiments is escalated (see Supplementary Fig. S3). The concept of a priming agent sensitizing leukemic cells to checkpoint inhibition has previously been described using oncolytic viruses [36] and STING agonists [35]. Our results further substantiate the beneficial effect of using an innate receptor agonist triggering an IFN-mediated immune activation and simultaneously blocking IFNregulated immune checkpoints like the PD-1/PD-L1 axis.

However, even the combination of PD-1 blockade and ppp-RNA treatment induced long-term remissions only in a fraction of mice in our study. A recent publication found a marked immunological heterogeneity even in s.c. tumor models derived from clonal cell lines that explained the differential response to immune checkpoint blockade even 
in inbred mouse strains [37]. A similar mechanism may explain the differences between responders and nonresponders in the AML model used here, where the disseminated AML cells may reach different niches, in which tumor cells are less susceptible to the treatment by pppRNA or the action of specific $\mathrm{T}$ cells. In addition, other stochastic processes may influence the establishment of additional immunosuppressive mechanisms other than the PD-1/PDL1 checkpoint. Better transfection reagents reaching those niches, higher doses of ppp-RNA, the combination with other cytotoxic strategies like chemo- or radiotherapy, inclusion of vaccine approaches e.g. based on tumor-mutation specific RNA applications (as described in Sahin et al. [38].) and additional checkpoint inhibitors, e.g. targeting CTLA4, LAG3 or TIM3, might therefore be required to increase the rate of long-term remissions.

To evaluate the potential of ppp-RNA based immunotherapy for clinical translation, we designed a humanized mouse model based on the idea of an allogeneic donor lymphocyte transfusion (DLI). DLI is a procedure that was initially established for relapsed chronic myeloid leukemia after SCT. Recent studies indicate a beneficial effect of DLI in patients suffering from relapsed high-risk AML after SCT [39, 40]. To simulate this setting, we infused human PDX AML-bearing NSG mice with allogeneic human PBMCs. The slow growth of PDX AML cells and looming GvH reactions in this xenograft model forced us to perform the PBMC infusion late, close to terminal stages of the disease and to use low numbers of transfused PBMCs. This left us a very small time window for treatment analysis. Nevertheless, we observed a reduction of tumor mass in the peripheral blood and bone marrow of ppp-RNA treated mice, while at the same time $\mathrm{CD}^{+} \mathrm{T}$ cell counts rose in the respective tissues, indicating a boosted $\mathrm{GvL}$ reaction triggered by ppp-RNA. More detailed studies will be needed to fully evaluate whether ppp-RNA treatment can potentiate the effects of DLI in relapsed AML patients after SCT or whether the effect is rather mediated by direct cytotoxic effects of ppp-RNA on the leukemic cells.

In summary, ppp-RNA treatment induces an immune cell-mediated response against disseminated AML, leads to long-term survival and establishes an immunological memory protective against leukemia relapse. In addition, treatment with ppp-RNA sensitizes tumors to therapeutic immune checkpoint blockade using an anti-PD-1 antibody.

Acknowledgements The authors thank Christine Hoerth and Natalie Roeder for technical support and Philipp Metzger and Sabrina Kirchleitner for advice on flow cytometry panel design. Furthermore, we thank Carolin Daniel from the Institute of Diabetes Research at the Helmholtz Zentrum München for support on the humanized mouse model. This work was supported by Einheit für Klinische Pharmakologie (EKLIP), Helmholtz Zentrum München, Neuherberg, Germany (to SR, FSL, SE, and JA), the international doctoral program "iTarget: Immunotargeting of cancer" funded by the Elite Network of Bavaria (to MS, LMK, SK, and SE), the Marie-Sklodowska-Curie Training Network for the Immunotherapy of Cancer (IMMUTRAIN, grant number 641549) funded by the H2020 program of the European Union (to SF, SE, SK and MS), the Friedrich-Baur-Stiftung and Else Kröner-Fresenius-Stiftung 2017_A50 to LMK, the Deutsche Forschungsgemeinschaft SCHN 664/ 6-1 and SFB 1321/P16 grant number 329628492 to MS, Ro 25257/-1 grant number 391217598 and SFB/TR-237-B14 grant number 404450088 to SR, the Metiphys fellowship of the Medical Faculty of the Ludwig-Maximilians-Universität München to FSL.

Author contribution MR designed and performed in vivo experiments, analyzed results, and co-wrote the paper. LMK and SR designed the research, supervised and analyzed experiments and cowrote the manuscript. FSL initiated the project, gave conceptual advice and co-wrote the manuscript. EH established the C1498 tumor model. SF, LK, JA, HM, MZ, EH, and DFRB performed experiments. BV and IJ provided the PDX AML model and advised on the humanized mouse model. MS, MaS, SK, PD, and SE gave conceptual advice. All authors reviewed the paper.

\section{Compliance with ethical standards}

Conflict of interest The authors declare no competing financial interests. Parts of this work have been performed for the doctoral theses of MR, EH, HM, and MZ at the LMU Munich.

Publisher's note Springer Nature remains neutral with regard to jurisdictional claims in published maps and institutional affiliations.

Open Access This article is licensed under a Creative Commons Attribution 4.0 International License, which permits use, sharing, adaptation, distribution and reproduction in any medium or format, as long as you give appropriate credit to the original author(s) and the source, provide a link to the Creative Commons license, and indicate if changes were made. The images or other third party material in this article are included in the article's Creative Commons license, unless indicated otherwise in a credit line to the material. If material is not included in the article's Creative Commons license and your intended use is not permitted by statutory regulation or exceeds the permitted use, you will need to obtain permission directly from the copyright holder. To view a copy of this license, visit http://creativecommons. org/licenses/by/4.0/.

\section{References}

1. Reinisch A, Chan SM, Thomas D, Majeti R. Biology and clinical relevance of acute myeloid leukemia stem cells. Semin Hematol. 2015;52:150-64.

2. Kassim AA, Savani BN. Hematopoietic stem cell transplantation for acute myeloid leukemia: a review. Hematol/Oncol Stem Cell Ther. 2017;10:245-51.

3. Lichtenegger FS, Krupka C, Haubner S, Köhnke T, Subklewe M. Recent developments in immunotherapy of acute myeloid leukemia. J Hematol Oncol. 2017;10:1-20.

4. Poeck H, Besch R, Maihoefer C, Renn M, Tormo D, Morskaya SS, et al. 5'-Triphosphate-siRNA: turning gene silencing and Rig-I activation against melanoma. Nat Med. 2008;14: 1256-63.

5. Tormo D, Checińska A, Alonso-Curbelo D, Pérez-Guijarro E, Cañón E, Riveiro-Falkenbach $\mathrm{E}$, et al. Targeted activation of innate immunity for therapeutic induction of autophagy and apoptosis in melanoma cells. Cancer Cell. 2009;16:103-14.

6. Ellermeier J, Wei J, Duewell P, Hoves S, Stieg MR, Adunka T, et al. Therapeutic efficacy of bifunctional siRNA combining TGF- 
$\beta 1$ silencing with RIG-I activation in pancreatic cancer. Cancer Res. 2013;73:1709-20.

7. Elion DL, Jacobson ME, Hicks DJ, Rahman B, Sanchez V, Gonzales-Ericsson PI, et al. Therapeutically active RIG-I agonist induces immunogenic tumor cell killing in breast cancers. Cancer Res. 2018;78:6183-95.

8. Hornung V, Ellegast J, Kim S, Brzózka K, Jung A, Kato H, et al. 5'-Triphosphate RNA is the ligand for RIG-I. Science. 2006;314:994-7.

9. Kato H, Sato S, Yoneyama M, Yamamoto M, Uematsu S, Matsui $\mathrm{K}$, et al. Cell type-specific involvement of RIG-I in antiviral response. Immunity. 2005;23:19-28.

10. Schlee M, Roth A, Hornung V, Hagmann CA, Wimmenauer V, Barchet W, et al. Recognition of $5^{\prime}$ triphosphate by RIG-I helicase requires short blunt double-stranded RNA as contained in panhandle of negative-strand virus. Immunity. 2009;31:25-34.

11. Schmidt A, Schwerd T, Hamm W, Hellmuth JC, Cui S, Wenzel $\mathrm{M}$, et al. 5'-triphosphate RNA requires base-paired structures to activate antiviral signaling via RIG-I. Proc Natl Acad Sci USA. 2009;106:12067-72.

12. Yoneyama M, Kikuchi M, Natsukawa T, Shinobu N, Imaizumi T, Miyagishi M, et al. The RNA helicase RIG-I has an essential function in double-stranded RNA-induced innate antiviral responses. Nat Immunol. 2004;5:730-7.

13. Kawai T, Takahashi K, Sato S, Coban C, Kumar H, Kato H, et al. IPS-1, an adaptor triggering RIG-I- and Mda5-mediated type I interferon induction. Nat Immunol. 2005;6:981-8.

14. Meylan E, Curran J, Hofmann K, Moradpour D, Binder M, Bartenschlager R, et al. Cardif is an adaptor protein in the RIG-I antiviral pathway and is targeted by hepatitis $\mathrm{C}$ virus. Nature. 2005;437:1167-72.

15. Seth RB, Sun L, Ea C-K, Chen ZJ. Identification and characterization of MAVS, a mitochondrial antiviral signaling protein that activates NF-кB and IRF3. Cell. 2005;122:669-82.

16. Chow KT, Gale M, Loo Y-M. RIG-I and other RNA sensors in antiviral immunity. Annu Rev Immunol. 2018;36:667-94.

17. Besch R, Poeck H, Hohenauer T, Senft D, Häcker G, Berking C, et al. Proapoptotic signaling induced by RIG-I and MDA-5 results in type I interferon-independent apoptosis in human melanoma cells. J Clin Investig. 2009;119:2399-411.

18. Duewell P, Steger A, Lohr H, Bourhis H, Hoelz H, Kirchleitner $\mathrm{SV}$, et al. RIG-I-like helicases induce immunogenic cell death of pancreatic cancer cells and sensitize tumors toward killing by CD8 (+) T cells. Cell Death Differ. 2014;21:1825-37.

19. Kübler K, Gehrke N, Riemann S, Böhnert V, Zillinger T, Hartmann E, et al. Targeted activation of RNA helicase retinoic acidinducible gene-I induces proimmunogenic apoptosis of human ovarian cancer cells. Cancer Res. 2010;70:5293-304.

20. Yuan D, Xia M, Meng G, Xu C, Song Y, Wei J. Anti-angiogenic efficacy of 5'-triphosphate siRNA combining VEGF silencing and RIG-I activation in NSCLCs. Oncotarget. 2015;6:29664-74.

21. Sun YW. Rig-I, a homolog gene of RNA helicase, is induced by retinoic acid during the differentiation of acute promyelocytic leukemia cell. Thesis, Shanhai Institute of Heamatology, Rui-Jin Hospital, Shanghai Second Medical University. Gene Bank accession number AF038963; 1997.

22. Li X-Y, Jiang L-J, Chen L, Ding M-L, Guo H-Z, Zhang W, et al. RIG-I modulates Src-mediated AKT activation to restrain leukemic stemness. Mol Cell. 2014;53:407-19.

23. Garcia-Diaz A, Shin DS, Moreno BH, Saco J, Escuin-Ordinas H, Rodriguez GA, et al. Interferon receptor signaling pathways regulating PD-L1 and PD-L2 expression. Cell Rep. 2017;19:1189-201.

24. Bald T, Landsberg J, Lopez-Ramos D, Renn M, Glodde N, Jansen $\mathrm{P}$, et al. Immune cell-poor melanomas benefit from PD-1 blockade after targeted type I IFN activation. Cancer Discov. 2014;4:674-87.

25. Gubin MM, Zhang X, Schuster H, Caron E, Ward JP, Noguchi T, et al. Checkpoint blockade cancer immunotherapy targets tumourspecific mutant antigens. Nature. 2014;515:577-81.

26. Robert C, Long GV, Brady B, Dutriaux C, Maio M, Mortier L, et al. Nivolumab in previously untreated melanoma without BRAF mutation. N Engl J Med. 2015;372:320-30.

27. Tumeh PC, Harview CL, Yearley JH, Shintaku IP, Taylor EJM, Robert L, et al. PD-1 blockade induces responses by inhibiting adaptive immune resistance. Nature. 2014;515:568-71.

28. Zhang L, Gajewski TF, Kline J. PD-1/PD-L1 interactions inhibit antitumor immune responses in a murine acute myeloid leukemia model. Blood. 2009;114:1545-52.

29. Vick B, Rothenberg M, Sandhöfer N, Carlet M, Finkenzeller C, Krupka C, et al. An Advanced preclinical mouse model for acute myeloid leukemia using patients' cells of various genetic subgroups and in vivo bioluminescence imaging. PLoS ONE. 2015;10:e0120925-20.

30. Mopin A, Driss V, Brinster C. A detailed protocol for characterizing the murine $\mathrm{C} 1498$ cell line and its associated leukemia mouse model. J Vis Exp. 2016. https://doi.org/10.3791/54270.

31. Schlee M, Hartmann G. Discriminating self from non-self in nucleic acid sensing. Nat Rev Immunol. 2016;16:566-80.

32. Ellermeier J. Immuntherapie des Pankreaskarzinoms: Mechanismen und Strategien zur Durchbrechung tumorinduzierter Immunsuppression. Elektronische Hochschulschriften der LMU, München. 2014;1-74. http://nbn-resolving.de/urn:nbn:de:bvb:19165221.

33. Hochheiser K, Klein M, Gottschalk C, Hoss F, Scheu S, Coch C, et al. Cutting edge: the RIG-I ligand 3pRNA potently improves CTL cross-priming and facilitates antiviral vaccination. J Immunol. 2016;196:1501958-2443.

34. Bourquin C, Hotz C, Noerenberg D, Voelkl A, Heidegger S, Roetzer LC, et al. Systemic cancer therapy with a small molecule agonist of toll-like receptor 7 can be improved by circumventing TLR tolerance. Cancer Res. 2011;71:5123-33.

35. Curran E, Chen X, Corrales L, Kline DE, Dubensky TW, Duttagupta $\mathrm{P}$, et al. STING pathway activation stimulates potent immunity against acute myeloid leukemia. Cell Rep. 2016;15:2357-66.

36. Shen W, Patnaik MM, Ruiz A, Russell SJ, Peng K-W. Immunovirotherapy with vesicular stomatitis virus and PD-L1 blockade enhances therapeutic outcome in murine acute myeloid leukemia. Blood. 2016;127:1449-58.

37. Zemek RM, De Jong E, Chin WL, Schuster IS, Fear VS, Casey $\mathrm{TH}$, et al. Sensitization to immune checkpoint blockade through activation of a STAT1/NK axis in the tumor microenvironment. Sci Transl Med. 2019;11:eaav7816.

38. Sahin U, Derhovanessian E, Miller M, Kloke B-P, Simon P, Löwer $\mathrm{M}$, et al. Personalized RNA mutanome vaccines mobilize poly-specific therapeutic immunity against cancer. Nature. 2017;547:222-6.

39. Schmid C, Labopin M, Nagler A, Bornhäuser M, Finke J, Fassas A, et al. Donor lymphocyte infusion in the treatment of first hematological relapse after allogeneic stem-cell transplantation in adults with acute myeloid leukemia: a retrospective risk factors analysis and comparison with other strategies by the EBMT Acute Leukemia Working Party. J Clin Oncol. 2007;25:4938-45.

40. Schmid C, Labopin M, Schaap N, Veelken H, Schleuning M, Stadler M, et al. Prophylactic donor lymphocyte infusion after allogeneic stem cell transplantation in acute leukaemia-a matched pair analysis by the Acute Leukaemia Working Party of EBMT. Br J Haematol. 2018;21:454-787. 